\title{
Evaluation of end-tidal carbon dioxide gradient as a predictor of volume responsiveness in spontaneously breathing healthy adults
}

\author{
María C. Arango-Granados ${ }^{1,2^{*}}$ (D) Virginia Zarama Córdoba ${ }^{2}$, Andrés M. Castro Llanos ${ }^{2}$ \\ and Luis A. Bustamante Cristancho ${ }^{2}$
}

\author{
* Correspondence: \\ arango.mc@gmail.com \\ 'Universidad Icesi, Calle $18 \mathrm{No}$ \\ 122-135 Pance, Cali, Colombia \\ ${ }^{2}$ Fundación Valle del Lili, Av. Simón \\ Bolívar. Cra 98 \# 18-49, Cali, \\ Colombia
}

\begin{abstract}
Background: Methods to guide fluid therapy in spontaneously breathing patients are scarce. No studies have reported the accuracy of end-tidal $\mathrm{CO}_{2}\left(\mathrm{ET}-\mathrm{CO}_{2}\right)$ to predict volume responsiveness in these patients. We sought to evaluate the ET- $\mathrm{CO}_{2}$ gradient $\left(\Delta \mathrm{ET}-\mathrm{CO}_{2}\right)$ after a passive leg rise (PLR) maneuver to predict volume responsiveness in spontaneously breathing healthy adults.

Methods: We conducted a prospective study in healthy adult human volunteers. A PLR maneuver was performed and cardiac output (CO) was measured by transthoracic echocardiography. ET-CO2 was measured with non-invasive capnographs. Volume responsiveness was defined as an increase in cardiac output (CO) $>12 \%$ at $90 \mathrm{~s}$ after PLR.

Results: Of the 50 volunteers, $32 \%$ were classified as volume responders. In this group, the left ventricle outflow tract velocity time integral (VTILVOT) increased from $17.9 \pm 3.0$ to $20.4 \pm 3.4(p=0.0004)$, $C O$ increased from $4.4 \pm 1.5$ to $5.5 \pm 1.6(p=0.0)$, and $E T-\mathrm{CO}_{2}$ rose from $32 \pm 4.84$ to $33 \pm 5.07(p=0.135)$. Within the entire population, PLR-induced percentage $\Delta \mathrm{CO}$ was not correlated with percentage $\Delta \mathrm{ET}-\mathrm{CO}_{2}\left(R^{2}=0.13 ; p=0.36\right)$. The area under the receiver operating curve for the ability of $\mathrm{ET}^{-} \mathrm{CO}_{2}$ to discriminate responders from non-responders was of $0.67 \pm 0.09$ ( $95 \% \mathrm{Cl} 0.498-0.853)$. A $\Delta \mathrm{ET}-\mathrm{CO}_{2}$ $\geq 2 \mathrm{mmHg}$ had a sensitivity of $50 \%$, specificity of $97.06 \%$, positive likelihood ratio of 17.00 , negative likelihood ratio of 0.51 , positive predictive value of $88.9 \%$, and negative predictive value of $80.5 \%$ for the prediction of fluid responsiveness.
\end{abstract}

Conclusions: $\triangle \mathrm{ET}-\mathrm{CO}_{2}$ after a PLR has limited utility to discriminate responders from non-responders among healthy spontaneously breathing adults.

Keywords: Capnography, Cardiac output, Doppler echocardiography, Blood volume determination, Hemodynamic monitoring

\section{Background}

One of the most important goals during the resuscitation of the critically ill patient is to reestablish an adequate oxygen delivery (DO2) to the tissues. Given the theory that DO2 depends, among others, on cardiac output (CO), the clinician may consider to give intravenous fluids aiming to increase left ventricular preload and stroke volume (SV). However, it is known that not every patient responds to a volume challenge with 
an increase in CO. Furthermore, fluid administration requires careful monitoring because both, volume deficit and overload, can worsen the prognosis $[1,2]$.

For this reason, the fundamental challenge is to accurately estimate if the patient will benefit from a volume challenge. Methods to guide fluid therapy in the spontaneously breathing patient are scarce, and many of them require invasive monitoring [3]. This may limit its routine use in patients who present to the emergency department or who are being treated in scenarios that do not have these monitoring tools within reach.

An interesting and non-invasive tool is the evaluation of the aortic flow by transthoracic echocardiography (TTE). Variables such as the change in peak aortic flow velocity and left ventricle outflow tract velocity time integral $\left(\mathrm{VTI}_{\mathrm{LVOT}}\right)$ after a passive leg rise (PLR) maneuver are good predictors of volume responsiveness in critically ill patients (sensitivity $77 \%$ and $100 \%$ specificity) $[4,5]$; however, this echocardiographic measurements require more advanced training, can be difficult to estimate in patients with inadequate acoustic windows, there are important limitations with keeping the same angle between probe and the left outflow tract in both positions, and finally, these measurements take a considerable time to obtain.

The relationship between expired $\mathrm{CO}_{2}\left(\mathrm{ET}-\mathrm{CO}_{2}\right)$ and $\mathrm{CO}$ has been known for several decades [6, 7]. Since ET- $\mathrm{CO}_{2}$ is determined mainly by the tissue production of carbon dioxide $\left(\mathrm{CO}_{2}\right)$, alveolar ventilation and $\mathrm{CO}[8]$, under constant metabolic and ventilatory conditions, acute changes in ET- $\mathrm{CO}_{2}$ have shown to correlate strongly with changes in $\mathrm{CO}$ in experimental [9-11] and clinical $[12,13]$ scenarios. For this reason, the variation in ET- $\mathrm{CO}_{2}$ has been used as a method to predict volume responsiveness after a PLR maneuver in the mechanically ventilated patient [14].

To date, there are no published studies evaluating the accuracy of non-invasive ET- $\mathrm{CO}_{2}$ to predict volume responsiveness in the spontaneously breathing patient. It is well known that healthy individuals can work at various points of the Frank-Starling curve at different times due to small changes in their cardiac contractility and/or effective vascular volume, so a percentage of them can respond to volume at a given moment [15]. Therefore, the objective of this study is to evaluate the performance of the expired $\mathrm{CO}_{2}$ gradient $\left(\triangle \mathrm{ET}-\mathrm{CO}_{2}\right)$ after a PLR maneuver to predict volume responsiveness in spontaneously breathing healthy adults.

\section{Methods}

\section{Study population}

We included human volunteers of both sexes, $\geq 18$ years, classified according to the American Society of Anesthesiologists as ASA I or ASA II. This classification (ASA I and II) includes healthy people or with mild systemic diseases without major functional limitations [16]. Volunteers who presented arrhythmias at the time of analysis, lower limb amputation, inadequate cardiac window for $\mathrm{VTI}_{\mathrm{LVOT}}$ measurement, pregnant women, participants who during the maneuver did not tolerate supine position or leg rise, and finally, patients who refused to participate were excluded.

A non-probability convenience sample was used, estimating a total of at least 50 participants. Volunteers were mainly medical school students, residents, hospital staff, and close contacts of the researchers (family, friends, and colleagues). This study was conducted in the Valle del Lili Foundation Hospital (Cali, Colombia) after approval by 
the Institutional Ethics Committee. All informed consents are duly signed and stored in this department.

\section{Measurements}

Participants were contacted individually and required to be fasted, according to the definition of fasting of the American Society of Anesthesiology [17]. In the laboratory, after 2 min of placing the participants on a supine position with head at $45^{\circ}$, baseline hemodynamic variables were recorded (Additional file 1: Table S1). Subsequently, the PLR maneuver was performed, according to the original description of the technique: semi recumbent position, total supine position with leg rise at $45^{\circ}$ for $90 \mathrm{~s}$, return to the basal position [18]. The different hemodynamic variables, including capnography, were recorded at 30, 60, 90, 5, 8, and 10 min after the initiation of the maneuver. $\operatorname{VTI}_{\mathrm{LVOT}}$ measurement was taken $90 \mathrm{~s}, 5 \mathrm{~min}$, and $10 \mathrm{~min}$ after the PLR.

For the purpose of this study, the participant who had an increase in $\mathrm{CO}>12 \%$ after $90 \mathrm{~s}$ of PLR was classified as a volume responder. $\mathrm{CO}$ was calculated by measuring the left ventricular outflow tract diameter (in the parasternal long axis view), the $\operatorname{VTI}_{\mathrm{LVOT}}$ (in the apical five-chamber view), and the heart rate $\left(\mathrm{CO}=\pi\left(\frac{D}{2}\right)^{2} \times \mathrm{VTI} \times \mathrm{HR}\right)$. All echocardiographic evaluations were performed by experienced sonographers (VZ, LB, CV).

Capnography was measured using Nihon Khoden cap-ONE ${ }^{\bullet}$ TG-920P mainstream $\mathrm{CO}_{2}$ sensor, attached to a disposable oral and nasal adaptor and placed directly at the point of expiration. The other hemodynamic variables and their source are described in Additional file 1: Table S1.

\section{Data management and statistical analysis}

An information quality control to determine missing data and extreme data was carried out. A Shapiro-Wilk test was used to determine the distribution of the numerical variables; these were summarized as mean and standard deviations or median and interquartile ranges, as appropriate. Categorical variables were summarized as proportions.

Differences between responders and non-responders were compared by means of an independent sample $t$ test, except for age, $\mathrm{SpO} 2, \mathrm{VTI}_{\mathrm{LVOT}}, \mathrm{SV}, \mathrm{CO}$, and cardiac index, which were compared by the Mann-Whitney $U$ test.

The effects of PLR on hemodynamic parameters were assessed using a paired Student's $t$ test. The area under the receiver operating curve (ROC) was calculated using the Hanley-McNeil test. ROC curves are presented as area \pm SE (95\% confidence interval). ET- $\mathrm{CO}_{2}$ 's capability to track changes in CO during PLR was tested using a concordance analysis through a Pearson correlation coefficient, both for percentage changes and for absolute values at each measuring point.

The best cutoff point was identified and reported in terms of sensitivity, specificity, positive predictive value (PPV), negative predictive value (NPV), positive likelihood ratio $(\mathrm{LR}+)$, and negative likelihood ratio (LR-). For this purpose, individuals with a decrease in ET-CO $\mathrm{CO}_{2}$ after PLR were assumed to have an increase of $0 \mathrm{mmHg}$. Trend graphs were constructed to evaluate the behavior of the hemodynamic variables over time (30 s-10 min). All statistical analyses were performed using STATA software. A $p$ value $<0.05$ was considered statistically significant. 


\section{Results}

A total of 50 volunteers were included in the final analysis. The flow of participants through the study is shown in Fig. 1. A large proportion of the population $(82 \%)$ had no past medical history; only 2 of them were hypertensive, 1 hypothyroid, 1 with sinus bradycardia, 2 with premature ventricular contractions, 2 with mild mitral regurgitation, 1 with a history of vasovagal syncope, and 1 with a history of stage 2 chronic kidney disease. The mean baseline $\mathrm{VTI}_{\mathrm{LVOT}}$ was $18.8 \pm 2.8 \mathrm{~cm}$, baseline $\mathrm{CO}$ was $4.3 \pm 1.1 \mathrm{~L} / \mathrm{min}$, and baseline ET- $\mathrm{CO}_{2}$ was $32.0 \pm 3.5 \mathrm{mmHg}$ (Table 1).

Of the 50 volunteers, a total of $16(32 \%)$ were classified as volume responders after the PLR. In this group of participants, the $\mathrm{VTI}_{\mathrm{LVOT}}$ increased from $17.9 \pm 3.01$ to $20.4 \pm 3.42(p=0.0004), \mathrm{CO}$ increased from $4.4 \pm 1.45$ to $5.5 \pm 1.57(p=0.00)$, and ET- $\mathrm{CO}_{2}$ rose from $32 \pm 4.84$ to $33 \pm 5.07(p=0.135)$ (Table 2$)$. In the group of non-responders, the $\mathrm{VTI}_{\mathrm{LVOT}}$ did not show significant changes (from $19.2 \pm 2.67$ to $19.3 \pm 2.33, p=0.636)$, nor did the CO $(4.3 \pm 0.99$ to $4.3 \pm 0.96, p=0.474)$ or the ET- $-\mathrm{CO}_{2}(32 \pm 2.76$ to $32 \pm 2.63, p=0.408)$ (Table 3$)$. Changes in $\mathrm{ET}-\mathrm{CO}_{2}, \mathrm{VTI}_{\mathrm{LVOT}}$, $\mathrm{HR}$, and $\mathrm{CO}$ throughout the PLR maneuver among responders and non-responders are shown in Figs. 2 and 3.

Within the entire population, the PLR-induced percentage change in $\mathrm{CO}$ was not correlated with changes in ET- $\mathrm{CO}_{2}\left(R^{2}=0.13 ; p=0.36\right)$ (Fig. 4a). There was also no correlation between PLR-induced percentage changes in $\mathrm{VTI}_{\mathrm{LVOT}}$ and ET- $\mathrm{CO}_{2}\left(R^{2}=\right.$ $0.18 ; p=0.21$ ) (Fig. 4b). Correlation between absolute values of $\mathrm{CO}$ and $\mathrm{ET}^{-\mathrm{CO}_{2}}$ at each measurement point (basal, $90 \mathrm{~s}, 5 \mathrm{~min}, 10 \mathrm{~min})$ were also non-significant $\left(R^{2}=\right.$ $0.03, p=0.82 ; R^{2}=0.18, p=0.20 ; R^{2}=0.11, p=0.43 ; R^{2}=0.10, p=0.48$, respectively) (Additional file 1: Figure S1).

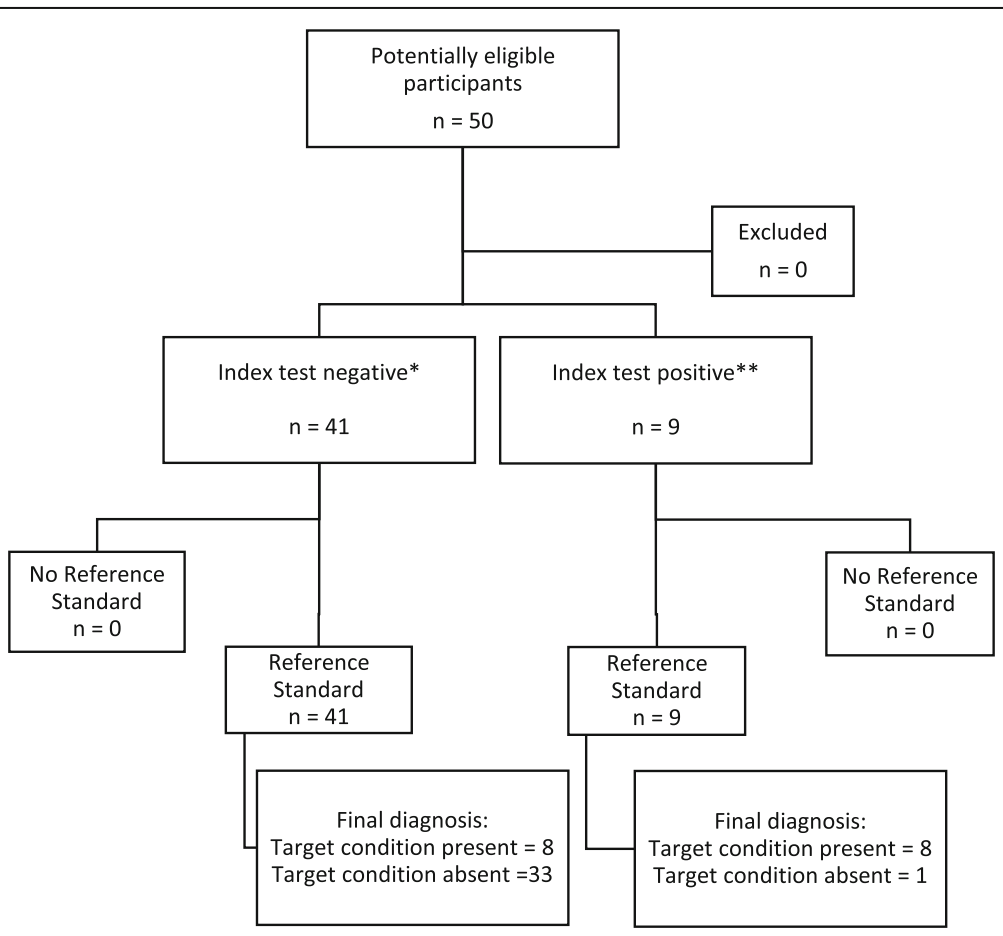

Fig. 1 Flow of participants through the study. ${ }^{*}$ Defined as $\triangle \mathrm{ET}-\mathrm{CO} 2<2 \mathrm{mmHg}$; ${ }^{* *}$ defined as $\triangle \mathrm{ET}-\mathrm{CO} 2 \geq 2 \mathrm{mmHg}$ 


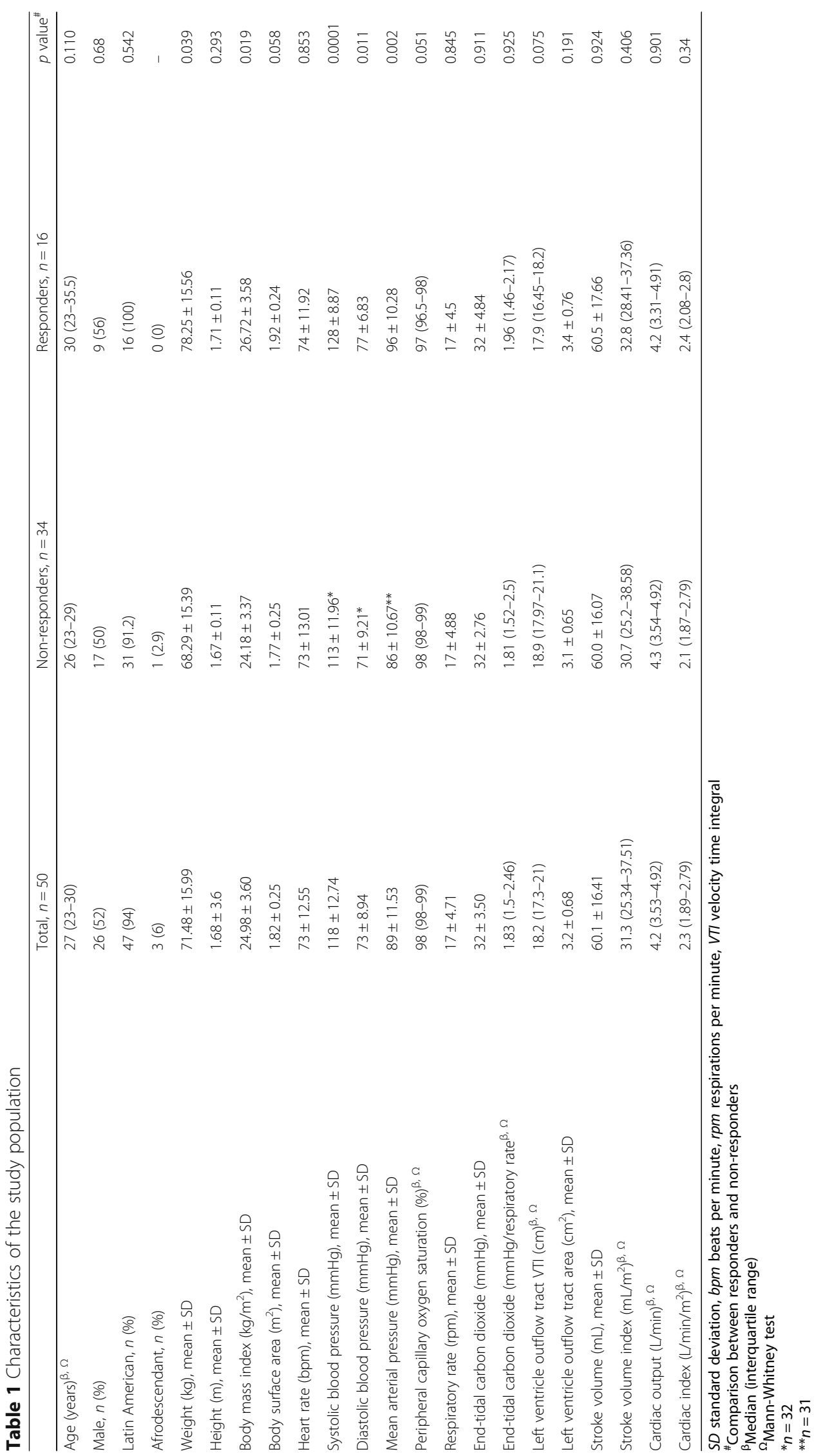




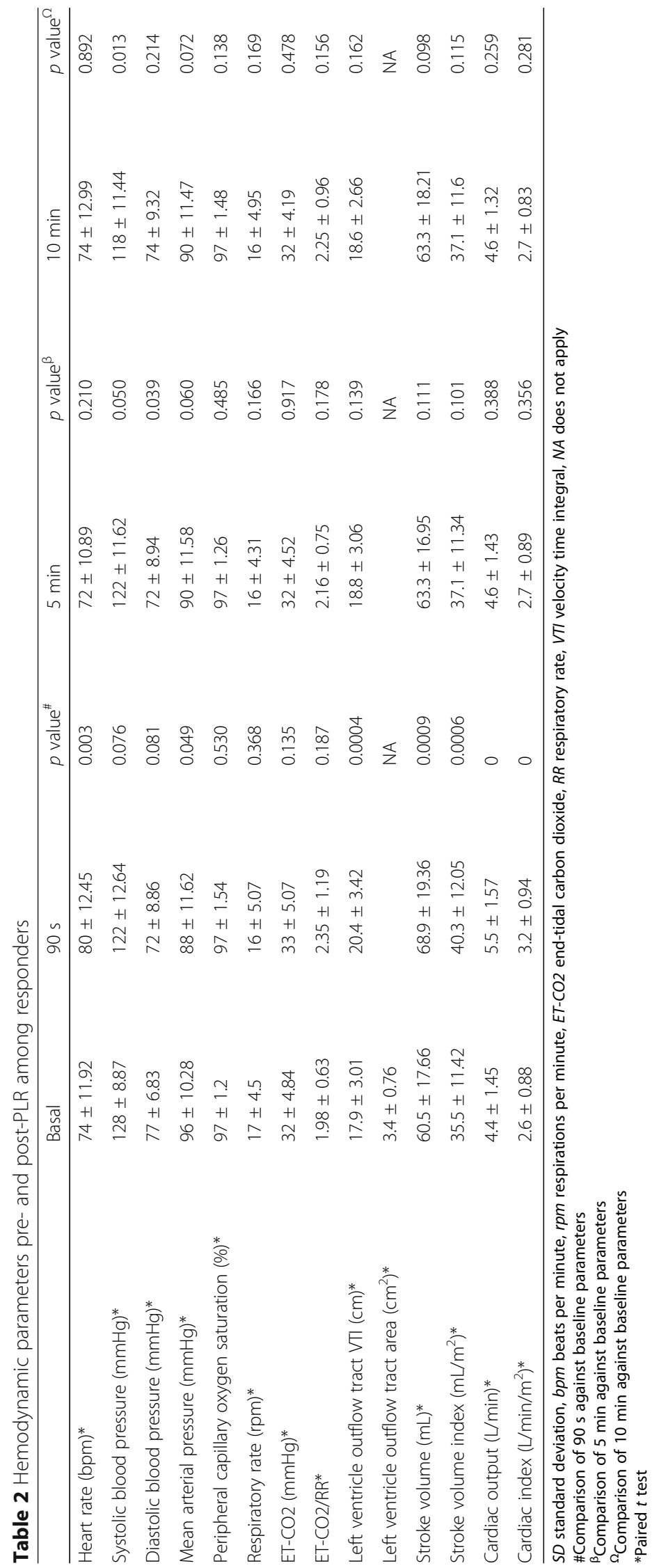




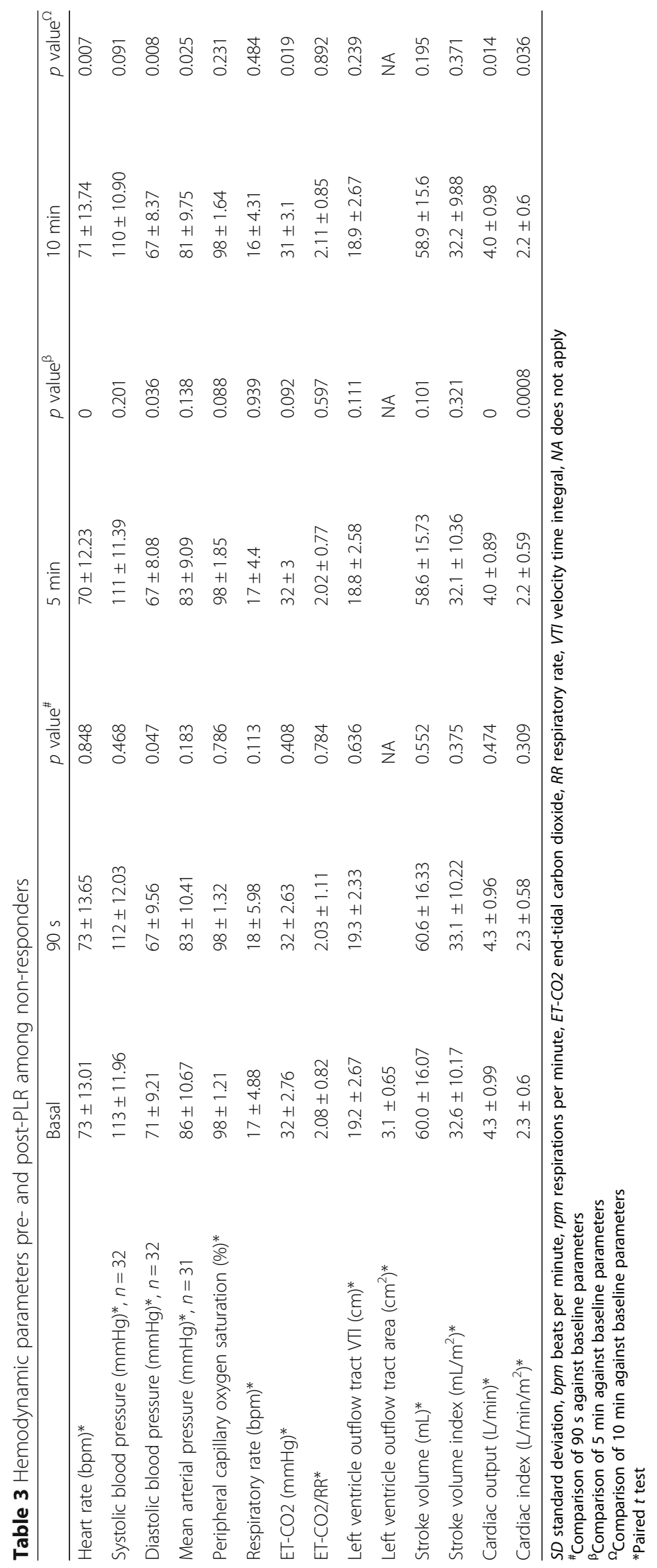




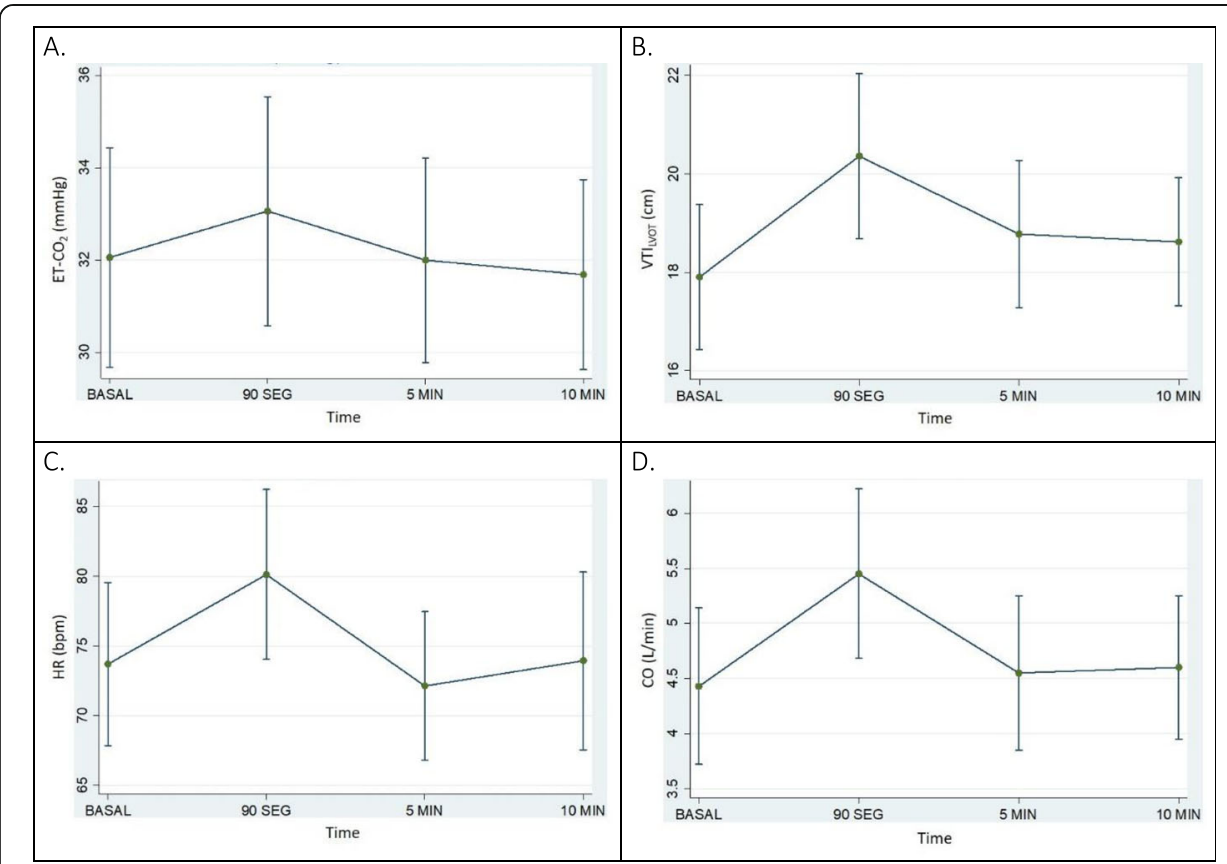

Fig. 2 Among responders, behavior during PLR maneuver of a ET-CO2, b VTILVOT, c HR, and d CO. ET-CO2, end-tidal carbon dioxide; $V \mathrm{VI}_{\mathrm{LVOT}}$, left ventricle outflow tract velocity time integral; $\mathrm{HR}$, heat rate; CO cardiac output

The area under the ROC curve for $\triangle \mathrm{ET}-\mathrm{CO}_{2}$ was of $0.67 \pm 0.09$ (95\% CI 0.498-0.853) (Fig. 5). A $\triangle \mathrm{ET}-\mathrm{CO}_{2} \geq 2 \mathrm{mmHg}$ had a sensitivity of $50 \%$, specificity of $97.06 \%$, LR + of 17.00, and LR- of 0.51 for the prediction of fluid responsiveness (Table 4). The PPV for this same cutoff point was $88.9 \%$ and the NPV was $80.5 \%$. The performance for the other cutoff points is shown in Table 4 . The performance analysis taking $\Delta \mathrm{VTI}_{\mathrm{LVOT}}$ as a gold standard is included in Additional file 1: Table S2.

In order not to ignore the probable effect of the heart rate in the determination of the $\mathrm{CO}$, trend curves throughout the PLR maneuver were constructed. In the group of responders, both the $\mathrm{VTI}_{\mathrm{LVOT}}$ and the $\mathrm{HR}$ (baseline $74 \pm 11.92$, $90 \mathrm{~s} 80 \pm 12.45, p=$ 0.0033 ) significantly increased from baseline to $90 \mathrm{~s}$. In the group of non-responders, although there was a slight but not significant rise in $\mathrm{VTI}_{\mathrm{LVOT}}$, HR remained unchanged (baseline $73 \pm 13.01$, 90 s $73 \pm 13.65, p=0.848$ ) (Table 3).

\section{Discussion}

According to the results of this study, the PLR-induced change in CO was not correlated with changes in ET- $\mathrm{CO}_{2}\left(R^{2}=0.13 ; p=0.36\right)$ in spontaneously breathing healthy adults. The area under the ROC curve for $\triangle \mathrm{ET}-\mathrm{CO}_{2}$ showed little utility of this measurement to discriminate responders from non-responders $(0.67 \pm 0.09$; $95 \%$ CI $0.498-0.853)$ (Fig. 5). Interestingly, a $\triangle \mathrm{ET}-\mathrm{CO}_{2} \geq 2 \mathrm{mmHg}$ had a specificity of $97.06 \%$, LR+ of 17.00, PPV of $88.9 \%$, and NPV of $80.5 \%$ for the prediction of fluid responsiveness (Table 4). This is consistent with the findings of Monge et al., who found that in mechanically ventilated patients with acute circulatory failure, an absolute increase in ET- $\mathrm{CO}_{2} \geq 2 \mathrm{mmHg}$ during PLR was associated with a positive response to fluid administration in all cases [14]. 


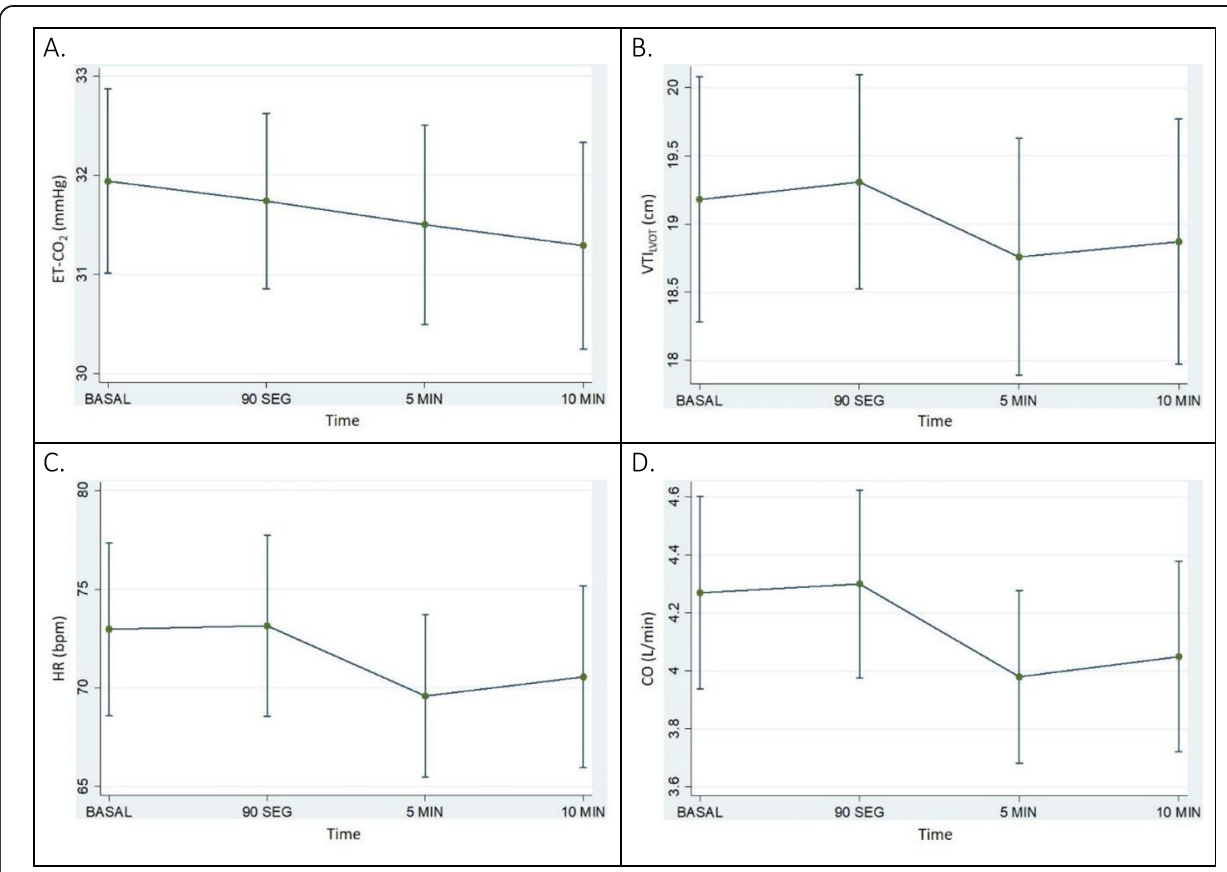

Fig. 3 Among non-responders, behavior during PLR maneuver of a ET-CO2, b VTILVOT, $\mathbf{c H R}$, and d CO. ET-CO2, end-tidal carbon dioxide; $\mathrm{VTI}_{\mathrm{LVOT}}$, left ventricle outflow tract velocity time integral; $\mathrm{HR}$, heat rate; $\mathrm{CO}$, cardiac output

To our knowledge, there is no study that has attempted to evaluate $\Delta \mathrm{ET}-\mathrm{CO}_{2}$ post-PLR to predict volume responsiveness in individuals under spontaneous breathing. In this subgroup, one of the most studied tests for this purpose is the ultrasonographic measurement of $\mathrm{CO}$ and its surrogates after a PLR maneuver. PLR provides a rapid and reversible 'self' volume challenge [18]. A meta-analysis published in 2010 found that this maneuver has a grouped sensitivity and specificity of 89.4 and $91.4 \%$, respectively. The threshold for the prediction of volume responsiveness varied within studies between 8 and 15\% [19]. However, although the heterogeneity according to the meta-analysis was not significant, studies included patients both ventilated and in spontaneous breathing. In addition, some studies used CO measurements as the gold standards, others used cardiac index, SV, or VTI $\mathrm{LVOT}_{\mathrm{LVO}}$. Some used TTE, others transesophageal echocardiography (TEE), minimally invasive $\mathrm{CO}$ monitoring through $\mathrm{PiCCO}^{\circ}$ or Vigileo/FloTrac ${ }^{\circ}$ systems, or invasive $\mathrm{CO}$ monitoring through pulmonary artery catheter.

Two studies performed on patients in spontaneous breathing and with echocardiographic measurements of $\mathrm{CO}$ should be highlighted. The first, published by Maizel et al., found that a change in $\mathrm{CO}>5 \%$ after a PLR discriminates responders from non-responders with a sensitivity of $94 \%$, specificity of $83 \%$, PPV of $83 \%$, and NPV of $94 \%$. In this same study, a change in $\mathrm{SV}>8 \%$ discriminated responders with a sensitivity of $88 \%$, specificity of $83 \%$, PPV of $82 \%$, and NPV of $88 \%$ [5]. The second study published by Lamia et al. found that a change in the $\operatorname{VTI}_{\mathrm{LVOT}}>12.5 \%$ discriminated responders with a sensitivity of $70 \%$ and specificity of $100 \%$ [4].

When evaluating stroke volume, Biais et al. found that a change in SV $>13 \%$ measured by TTE after PLR had a sensitivity of $100 \%$ and specificity of $80 \%$ to 


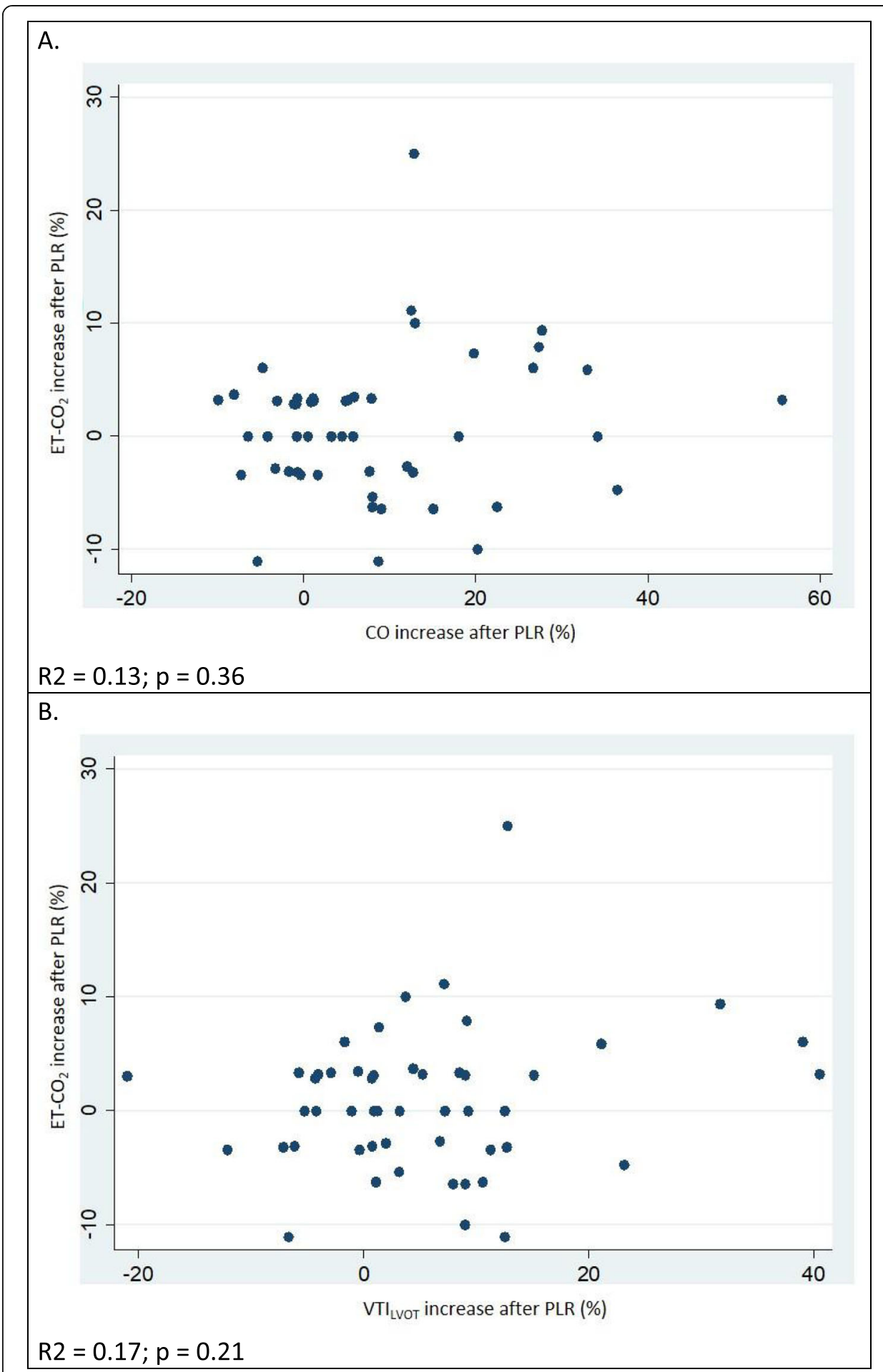

Fig. 4 Correlation between PLR-induced changes in ET-CO 2 and $\mathbf{a} \mathrm{CO}$ and $\mathbf{b} \mathrm{VTI}$ LVOT. ET-CO2, end-tidal carbon dioxide; $\mathrm{VTI}_{\mathrm{LVOT}}$, left ventricle outflow tract velocity time integral; $\mathrm{CO}$, cardiac output

predict volume responsiveness [20]. On their side, Préau et al. found that in patients with severe pancreatitis or sepsis, a change in SV $>10 \%$ had a sensitivity, specificity, PPV, and NPV of $86,90,86$, and $90 \%$, respectively [21]. 


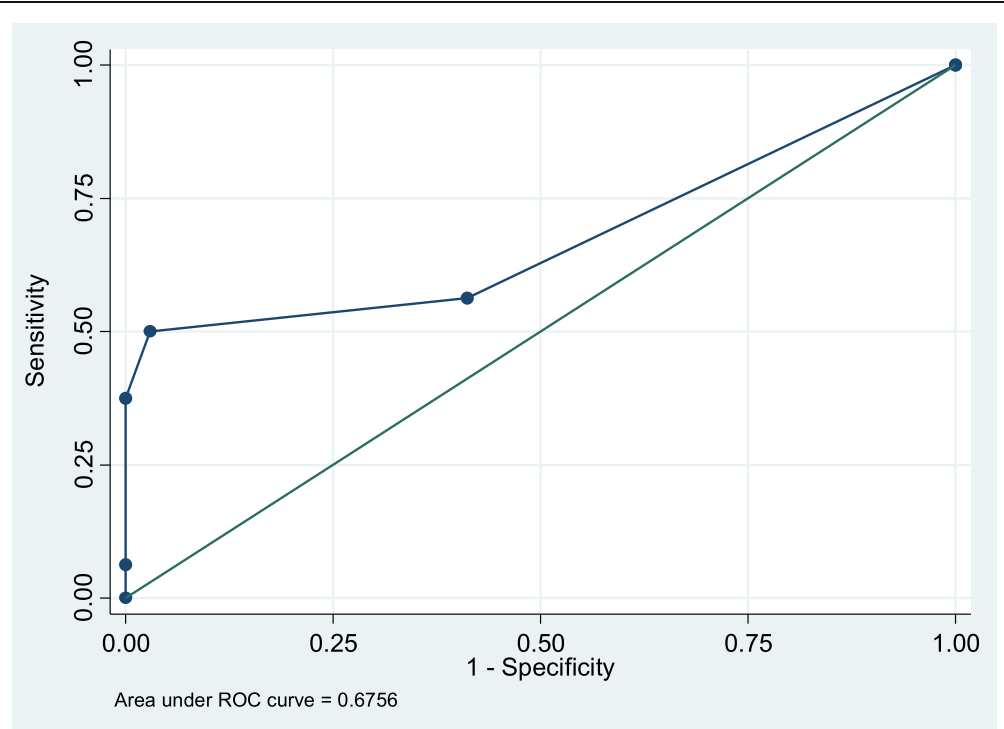

Fig. 5 Receiver operating characteristics curves regarding the ability of ET-CO $-\mathrm{CO}_{2}$ to discriminate responders (CO increase $\geq 12 \%$ ) and non-responders after a PLR maneuver. ET-CO2, end-tidal carbon dioxide; CO, cardiac output; PLR, passive leg rise

However, the acoustic window for optimal aortic flow Doppler alignment in critically ill patients and the technical difficulty to keep the same probe angle during the PLR maneuver make this measurement not always feasible in the daily clinical practice.

The results of this study suggest that $\triangle \mathrm{ET}-\mathrm{CO}_{2}$ after a PLR maneuver lack utility to predict volume responsiveness among healthy adult individuals. However, some considerations must be taken into account. First, although echocardiography is validated for $\mathrm{CO}$ determination, with $\mathrm{VTI}_{\mathrm{LVOT}}$ as the variable with less interobserver variability, it is not free from limitations due to angulation and beam alignment difficulties [22]. This may explain why ET- $\mathrm{CO}_{2}$ has shown better correlation with invasive measurements [12, 13] rather than ultrasonographic estimations of $\mathrm{CO}$ [14].

Second, there was a concern about the possible influence of heart rate when defining an individual as volume responder, so trend curves throughout the PLR maneuver were constructed. Within responders, both the $\operatorname{VTI}_{\mathrm{LVOT}}$ (baseline $17.9 \pm 3.01,90 \mathrm{~s} 20.4 \pm 3.42$, $p=0.0004$ ) and the heart rate (basal $74 \pm 11.92$, $90 \mathrm{~s} 80 \pm 12.45 ; p=0.003$ ) had a statistically significant increase from baseline to $90 \mathrm{~s}$. In contrast, within non-responders, although there was a slight but not significant increase in $\operatorname{VTI}_{\text {LVOT }}$ (basal $19.2 \pm 2.67$,

Table 4 Performance of $\triangle E T-C O 2$ post-PLR against $\triangle C O \geq 12 \%$ for the prediction of volume responsiveness

\begin{tabular}{llllll}
\hline Cut-point $(\mathrm{mmHg})$ & Sensitivity (\%) & Specificity (\%) & Correctly classified (\%) & LR+ & LR- \\
\hline$\geq 0$ & 100.00 & 0.00 & 32 & 1.00 & NA \\
$\geq 1$ & 56.25 & 58.82 & 58 & 1.37 & 0.74 \\
$\geq 2$ & 50.00 & 97.06 & 82 & 17.00 & 0.51 \\
$\geq 3$ & 37.5 & 100.00 & 80 & NA & 0.62 \\
$\geq 6$ & 6.25 & 100.00 & 70 & NA & 0.94 \\
$>6$ & 0.00 & 100.00 & 68 & NA & 1.00 \\
\hline LR likelihood ratio, NA does not apply & & & &
\end{tabular}


90 s $19.3 \pm 2.33, p=0.636$ ), HR remained unchanged (baseline $73 \pm 13.01$, $90 \mathrm{~s} 73 \pm$ $13.65, p=0.848$ ) (Table 3).

Finally, despite the fact that the $\triangle \mathrm{ET}-\mathrm{CO}_{2}$ had poor correlation with $\mathrm{CO}$, and the ROC suggested limited utility to discriminate responders from non-responders, interestingly a $\triangle \mathrm{ET}-\mathrm{CO}_{2} \geq 2 \mathrm{mmHg}$ had a specificity of $97.06 \%$, LR + of 17.00 , PPV of $88.9 \%$, and NPV of $80.5 \%$ for the prediction of fluid responsiveness (Table 4). This may be due to the characteristics of the study population. It is known that healthy adults can respond with an increase in CO or SV after a "hed-down tilt" maneuver [23]. However, Parker et al. described how in the healthy human, at rest and in supine decubitus, the ventricular function curve is at its maximum with an end diastolic pressure of the left ventricle of approximately $10 \mathrm{mmHg}$. Below this point, there is a strong direct relationship between filling pressure and cardiac performance, while with higher filling pressures a plateau occurs. Therefore, in the supine position, the normal heart is usually not in the steep part of the ventricular function curve, but is in a unique position in which the cardiac output is possibly controlled by factors other than the filling pressures of the heart [24]. Probably, in acute and critical illness, there is greater variability in the individual positions among the ventricular function curve, and there is therefore greater probability to induce a change in $\mathrm{CO}$ after a volume challenge, as has already been described in mechanically ventilated patients with acute circulatory failure [14].

This study has some limitations that have to be accounted. First, because of the inherent limitations of echocardiography due to angulation and beam alignment difficulties [22], ET- $\mathrm{CO}_{2}$ has shown better correlation with invasive measurements $[12,13]$ rather than ultrasonographic estimations of CO [14]. However, recent studies have demonstrated a significant correlation $(r=0.95 ; p<0.0001)$ between TTE and pulmonary artery catheter (PAC) CO measurement, with a median bias of $0.2 \mathrm{~L} / \mathrm{min}$, limits of agreement between 1.3 and $1.8 \mathrm{~L} / \mathrm{min}$ and a precision of $9 \%$ for TTE (vs $8 \%$ for PAC) [25]. This way we felt confident to avoid an invasive method for the estimation $\mathrm{CO}$ in healthy volunteers.

Second, during spontaneous breathing, measurement of $\mathrm{ET}-\mathrm{CO}_{2}$ by direct or lateral capnography is limited by the inevitable air leak from the system, and the technique has low sensitivity to detect hypoventilation in sedated patients [26]. However, in patients who are not under the effects of sedation, direct or lateral capnographs coupled to nasal cannula have good diagnostic performance [27-29], which may even be comparable with capnography in patients on mechanical ventilation [30].

Third, for the echocardiographic estimation of $\mathrm{CO}$ there is an important difficulty when trying to keep the same angle between the probe and the LVOT in both semi recumbent and leg raised positions. For the purpose of this study, we did not opt for the mathematical correction of the effects of angulation, but tried to optimize the angulation of the transducer to make it as parallel to the flow as possible. Finally, the technical difficulties of performing these measurements in individuals with inadequate acoustic windows cannot be ignored, although it was not necessary to exclude any participant for this reason. All ultrasonographic measurements were made by experienced sonographers (VZ, LB, CV).

\section{Conclusions}

According to the results of this study, the performance of $\triangle \mathrm{ET}-\mathrm{CO}_{2}$ for the prediction of volume responsiveness in spontaneously breathing healthy adults revealed a sensitivity 
of $50 \%$, specificity of $97.06 \%$, LR + of 17.00 , LR- of 0.51 , PPV of $88.9 \%$, and NPV of $80.5 \%$ for a $\triangle \mathrm{ET}-\mathrm{CO}_{2} \geq 2 \mathrm{mmHg}$. The area under the ROC curve for $\triangle \mathrm{ET}-\mathrm{CO}_{2}$ was of $0.67 \pm 0.09$ (95\% CI 0.48-0.85), suggesting limited utility of this measurement to discriminate responders from non-responders. PLR-induced changes in $\mathrm{CO}$ were not correlated with changes in ET- $\mathrm{CO}_{2}\left(R^{2}=0.13 ; p=0.36\right)$ in spontaneously breathing healthy adults.

\section{Additional file}

Additional file 1: Table S1. Hemodynamic variables and source of origin. Table S2. Performance of $\triangle E T-C O 2$ post-PLR against $\triangle V T T \geq 12.5 \%$ for the prediction of volume responsiveness. Figure S1. Correlation between absolute values of ET-CO2 and CO at (A) baseline (B) $90 \mathrm{~s}$ (C) $5 \mathrm{~min}$ and (D) $10 \mathrm{~min}$ after a PLR maneuver. (DOCX 177 kb)

\section{Abbreviations}

CO: Cardiac output; CVP: Central venous pressure; ET- $\mathrm{CO}_{2}$ : End-tidal $\mathrm{CO}_{2}$; IVC: Inferior vena cava; PLR: Passive leg rise; SV: Stroke volume; TEE: Transesophageal echocardiography; TTE: Transthoracic echocardiography; VTILVOT: Left ventricle outflow tract velocity time integral; $\triangle \mathrm{ET}-\mathrm{CO}_{2}$ : End-tidal $\mathrm{CO}_{2}$ gradient

\section{Acknowledgements}

Special acknowledgement is given to Dr. Carlos Vesga (CV), cardiologist and echocardiographist of the Valle del Lili Foundation Hospital, for his contributions to the data acquisition.

\section{Funding}

This study was chosen to be sponsored by the Clinical Research Center of the Valle del Lili Foundation Hospital. This institution carried out the statistical analysis of the data, but did not participate in the decision of the devices to be used or bought for its development, nor in the writing of the final manuscript.

\section{Availability of data and materials}

The datasets supporting the conclusions of this article are included within the article and its additional files.

\section{Authors' contributions}

MCA conceived of the study and participated in the design, created the database, contributed to the data acquisition, and wrote the paper. This author is the guarantor of the paper and takes responsibility for the integrity of the work as a whole. MCA, VZ, and LAB participated in the study design. VZ and LAB performed echocardiographic assessments. All authors helped in the drafting of the work or revised it critically for important intellectual content, read and approved the final manuscript. Finally, all the authors are accountable for all aspects of the work in ensuring that questions related to the accuracy or integrity of any part of the work are appropriately investigated and resolved.

\section{Ethics approval and consent to participate}

This research is in line with the international recommendations on human research, the Nuremberg code, the Helsinki agreement, and the CIOM guidelines. This study was approved by the Institutional Ethics Committee of the Fundación Valle del Lili Hospital (approval letter no. 611-2017). The study protocol is registered in this department under the number 1184.

\section{Consent for publication}

All informed consents are duly signed and stored in the Institutional Ethics Committee of the Fundación Valle del Lili Hospital (Cali-Colombia).

\section{Competing interests}

The authors declare that they have no competing interests.

\section{Publisher's Note}

Springer Nature remains neutral with regard to jurisdictional claims in published maps and institutional affiliations.

Received: 23 May 2018 Accepted: 18 July 2018

Published online: 30 July 2018

\section{References}

1. Rosenberg AL, Dechert RE, Park PK, Bartlett RH, Network NNA (2009) Review of a large clinical series: association of cumulative fluid balance on outcome in acute lung injury: a retrospective review of the ARDSnet tidal volume study cohort. J Intensive Care Med 24(1):35-46

2. Prowle JR, Echeverri JE, Ligabo EV, Ronco C, Bellomo R (2010) Fluid balance and acute kidney injury. Nat Rev Nephrol 6(2):107-115

3. Mackenzie DC, Noble VE (2014) Assessing volume status and fluid responsiveness in the emergency department. Clin Exp Emerg Med 1(2):67-77

4. Lamia B, Ochagavia A, Monnet X, Chemla D, Richard C, Teboul UL (2007) Echocardiographic prediction of volume responsiveness in critically ill patients with spontaneously breathing activity. Intensive Care Med 33(7):1125-1132 
5. Maizel J, Airapetian N, Lorne E, Tribouilloy C, Massy Z, Slama M (2007) Diagnosis of central hypovolemia by using passive leg raising. Intensive Care Med 33(7):1133-1138

6. Leigh MD, Jenkins LC, Belton MK, Lewis GB (1957) Continuous alveolar carbon dioxide analysis as a monitor of pulmonary blood flow. Anesthesiology 18(6):878-882

7. Weil MH, Bisera J, Trevino RP, Rackow EC (1985) Cardiac output and end-tidal carbon dioxide. Crit Care Med 13(11):907-909

8. West JB (1977) State of the art: ventilation-perfusion relationships. Am Rev Respir Dis 116(5):919-943

9. Ornato JP, Garnett AR, Glauser FL (1990) Relationship between cardiac output and the end-tidal carbon dioxide tension. Ann Emerg Med 19(10):1104-1106

10. Dubin A, Murias G, Estenssoro E et al (2000) End-tidal CO2 pressure determinants during hemorrhagic shock. Intensive Care Med 26(11):1619-1623

11. Idris AH, Staples ED, O'Brien DJ et al (1994) End-tidal carbon dioxide during extremely low cardiac output. Ann Emerg Med 23(3):568-572

12. Shibutani K, Muraoka M, Shirasaki S, Kubal K, Sanchala VT, Gupte P (1994) Do changes in end-tidal PCO2 quantitatively reflect changes in cardiac output? Anesth Analg 79(5):829-833

13. Wahba RW, Tessler MJ, Béïque F, Kleiman SJ (1996) Changes in PCO2 with acute changes in cardiac index. Can J Anaesth 43(3):243-245

14. Monge García MI, Gil Cano A, Gracia Romero M, Monterroso Pintado R, Pérez Madueño V, Díaz Monrové JC (2012) Non-invasive assessment of fluid responsiveness by changes in partial end-tidal $\mathrm{CO} 2$ pressure during a passive leg-raising maneuver. Ann Intensive Care. https://doi.org/10.1186/2110-5820-2-9

15. Alves DR, Ribeiras R (2017) Does fasting influence preload responsiveness in ASA 1 and 2 volunteers? Braz J Anesthesiol 67(2):172-179

16. Ament R (1979) Origin of the ASA classification. Anesthesiology 51(2):179

17. Committee ASOA (2011) Practice guidelines for preoperative fasting and the use of pharmacologic agents to reduce the risk of pulmonary aspiration: application to healthy patients undergoing elective procedures: an updated report by the American Society of Anesthesiologists Committee on Standards and Practice Parameters. Anesthesiology 114(3):495-511

18. Monnet X, Teboul JL (2015) Passive leg raising: five rules, not a drop of fluid! Crit Care. https://doi.org/10.1186/ s13054-014-0708-5

19. Cavallaro F, Sandroni C, Marano C et al (2010) Diagnostic accuracy of passive leg raising for prediction of fluid responsiveness in adults: systematic review and meta-analysis of clinical studies. Intensive Care Med 36(9):1475-1483

20. Biais M, Vidil L, Sarrabay P, Cottenceau V, Revel P, Sztark F (2009) Changes in stroke volume induced by passive leg raising in spontaneously breathing patients: comparison between echocardiography and Vigileo/FloTrac device. Crit Care 13(6):R195

21. Préau S, Saulnier F, Dewavrin F, Durocher A, Chagnon $J L$ (2010) Passive leg raising is predictive of fluid responsiveness in spontaneously breathing patients with severe sepsis or acute pancreatitis. Crit Care Med 38(3):819-825

22. Lewis JF, Kuo LC, Nelson JG, Limacher MC, Quinones MA (1984) Pulsed Doppler echocardiographic determination of stroke volume and cardiac output: clinical validation of two new methods using the apical window. Circulation 70(3):425-431

23. Nixon JV, Murray RG, Leonard PD, Mitchell JH, Blomqvist CG (1982) Effect of large variations in preload on left ventricular performance characteristics in normal subjects. Circulation 65(4):698-703

24. Parker JO, Case RB (1979) Normal left ventricular function. Circulation 60(1):4-12

25. Mercado P, Maizel J, Beyls C et al (2017) Transthoracic echocardiography: an accurate and precise method for estimating cardiac output in the critically ill patient. Crit Care 21(1):136

26. Mehta JH, Williams GW, Harvey BC, Grewal NK, George EE (2017) The relationship between minute ventilation and end tidal $\mathrm{CO} 2$ in intubated and spontaneously breathing patients undergoing procedural sedation. PLoS One 12(6):e0180187

27. Casati A, Gallioli G, Passaretta R, Scandroglio M, Bignami E, Torri G (2001) End tidal carbon dioxide monitoring in spontaneously breathing, nonintubated patients. A clinical comparison between conventional sidestream and microstream capnometers. Minerva Anestesiol 67(4):161-164

28. Kasuya Y, Akça O, Sessler DI, Ozaki M, Komatsu R (2009) Accuracy of postoperative end-tidal Pco2 measurements with mainstream and sidestream capnography in non-obese patients and in obese patients with and without obstructive sleep apnea. Anesthesiology 111(3):609-615

29. Sakata DJ, Matsubara I, Gopalakrishnan NA et al (2009) Flow-through versus sidestream capnometry for detection of end tidal carbon dioxide in the sedated patient. J Clin Monit Comput 23(2):115-122

30. Casati A, Gallioli G, Scandroglio M, Passaretta R, Borghi B, Torri G (2000) Accuracy of end-tidal carbon dioxide monitoring using the NBP-75 microstream capnometer. A study in intubated ventilated and spontaneously breathing nonintubated patients. Eur J Anaesthesiol 17(10):622-626 